\title{
Ordered Hydrogen Bonding Structure of Water Molecules Adsorbed on Silver lodide Particles under Subsaturated Conditions
}

\author{
Huanyu Yang, Anthony Boucly, Jérôme Philippe Gabathuler, Thorsten Bartels-Rausch, Luca Artiglia,
} and Markus Ammann*

Cite This: J. Phys. Chem. C 2021, 125, 11628-11635

Read Online

\section{ACCESS I}

山lll Metrics \& More

回国 Article Recommendations

Supporting Information

ABSTRACT: Silver iodide (AgI) is an efficient ice-nucleating material. This has been related to the close lattice match to hexagonal ice, which helps to nucleate ice crystals on its surface under supersaturated conditions. In turn, the structure of water molecules adsorbed on its surface, embodied in the coordination of hydrogen bonds, has not been addressed so far. We suspected that AgI may induce ice-like coordination among adsorbed water molecules already under subsaturated conditions. X-ray photoelectron spectroscopy was used to probe the structure and composition at the AgI surface. We determined the chemical properties of the surface, the thickness of adsorbed water, and the

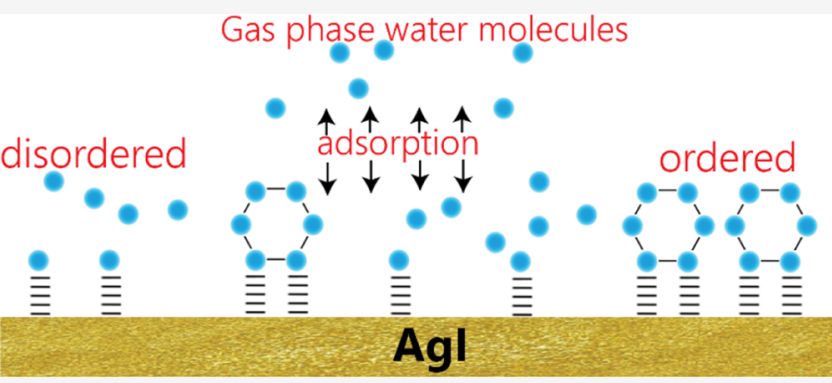
amount of contaminating carbon species. Auger electron yield near-edge X-ray absorption fine structure (NEXAFS) spectroscopy was used to assess the hydrogen bonding (HB) structure. The O K-edge NEXAFS spectra indicated that the HB structure of the adsorbed water on AgI under subsaturated conditions showed similarity to that of ice, which helps facilitate the stabilization of ice embryos at saturation. The approach used here opens up important perspectives for characterizing adsorbed water molecules on a wide variety of solids, which provides an important basis for understanding ice nucleation and other interfacial processes at the molecular level.

\section{INTRODUCTION}

Ice nucleation and ice particles are exerting a substantial impact on various physical and chemical processes of the atmosphere. For instance, cirrus ice clouds are important for the radiative balance of the atmosphere; ${ }^{1}$ the freezing of cloud water is crucial for the initiation of precipitation and influences cloud electrification; ${ }^{2}$ ice in clouds or on the ground promotes heterogeneous chemistry. ${ }^{3}$ Ice nucleation can occur homogeneously or heterogeneously. Homogeneous nucleation of ice in the gas phase or in pure liquid water is an activated process with a high free energy barrier for the stabilization of a critical ice cluster, the so-called ice embryo, requiring temperatures far below the melting point or high water vapor supersaturation. ${ }^{4}$ However, the atmosphere contains a multitude of condensed materials, which can stabilize an ice embryo and thus initiate ice nucleation at much higher temperatures and lower supersaturation. This is referred to as heterogeneous ice nucleation. It is the dominant mechanism by which ice forms in mixed phase clouds or by which cirrus ice particles form. ${ }^{5}$ Deposition ice nucleation is an important example of heterogeneous nucleation, in which ice nucleation of water molecules is initiated by the growth of the ice embryo on the surface of a solid material without the occurrence of liquid water. $^{6,7}$
In deposition ice nucleation, the interaction between the solid substrate and water molecules on its surface, controlled by the chemical composition and physical structure of the substrate, can lead to ordering of the hydrogen bonding (HB) network among these water molecules in a way to facilitate the growth and stabilization of ice embryos. ${ }^{8}$ In a similar way, in immersion freezing, the substrate influences the molecular orientation of water molecules near the solid-liquid interface, changing their interaction with neighboring water molecules in the bulk liquid and thus resulting in a different structure or density near the solid surface than in the bulk. ${ }^{9}$

Over the past decades, many groups implemented computational simulations ${ }^{10-15}$ and experimental studies ${ }^{16-18}$ to investigate the interfacial water structure on solid surfaces. Vonnegut ${ }^{19}$ attributed the high ice nucleation ability of AgI to the close lattice similarity between ice and AgI. Zielke et al. ${ }^{20}$ found that the AgI surface can impose a structure in the contact water layer close to that of bulk ice. Pedevilla et al. ${ }^{21}$

Received: February 26, 2021

Revised: April 28, 2021

Published: May 21, 2021 
found that at low coverage, single water molecules can bind strongly to the microcline feldspar's surface and in spite of the non-ice like structure of this layer, the longer range interactions lead to an ice-like structure in the layers beyond the first. Recently, surface-sensitive nonlinear optical spectroscopy was used to probe the interfacial water structure in relation to the ice nucleating (IN) ability of mineral surfaces. ${ }^{22-24}$ These studies also identified feedback between the water structure, surface charge, and ion dissolution on these surfaces. By using Kelvin Probe microscopy and in situ X-ray spectroscopy, Verdaguer et al. found the local structure of adsorbed water molecules on silica similar to ice at $70 \%$ relative humidity $(\mathrm{RH})$ at a temperature lower than $0{ }^{\circ} \mathrm{C}$ but liquid-like just above $0{ }^{\circ} \mathrm{C} .{ }^{25}$ Through computer simulation, $\mathrm{Hu}$ and Michaelides ${ }^{11}$ identified a 2D ice-like layer of water molecules on a kaolinite surface, attributed to the amphoteric property (ability to accept and donate hydrogen bonds) of kaolinite. In contrast, Qiu et al. ${ }^{13}$ found that water on carbon surfaces is similar to that in bulk liquid by using the monoatomic water model $\mathrm{mW}$. Even though the water structure is related to hydrophilic or hydrophobic properties of a surface, Lupi and Molinero $^{12}$ argued that the hydrophilicity of a surface in general is not a good predictor for heterogeneous ice nucleation.

Among the solids discussed above, AgI possesses an exceptional heterogeneous ice nucleation ability; ${ }^{19,26}$ it is widely used as an effective ice nucleus including the use as a cloud seeding material for artificial rain making. ${ }^{27}$ As already mentioned, many studies attributed the high ice nucleation ability of AgI to its lattice structure, which promotes the formation of ice crystals both in super cooled water and in water vapor super saturated with respect to ice. ${ }^{19}$ The unit cells of ice and silver iodide are both hexagonal and their lattice constants match within $98 \% .^{28,29}$ However, lattice match does not seem to be the only condition to explain the good ice nucleation ability of AgI. For instance, compounds with a lattice structure similar to that of ice, such as $\mathrm{BaF}_{2}{ }^{30}$ or quartz, $^{26}$ are inefficient IN materials. Conversely, organic compounds such as steroids have no structural similarity with ice but show an outstanding IN ability. ${ }^{31}$ The contrast between $\mathrm{AgI}$ and $\mathrm{BaF}_{2}$ may be resolved by considering their solubility in water, and the effective charge of ions of these ionic crystals. $\mathrm{BaF}_{2}$ is a strongly ionic compound ${ }^{32}$ with a solubility in water of $1.6 \mathrm{~g} / \mathrm{L}$; the effective charge of $\mathrm{Ba}$ is $1.8 \mathrm{eV}$ and that of $\mathrm{F}$ is $-0.8 \mathrm{eV}$. The strong electric field arising from the two ions may inhibit the water molecules on the surface to orient in an ice-like structure. ${ }^{30}$ Conversely, AgI has a solubility lower by 6 orders of magnitude in water than that of $\mathrm{BaF}_{2}{ }^{30,33}$ (about 3 . $10^{-6} \mathrm{~g} / \mathrm{L}$ ) and is considered hydrophobic. ${ }^{26}$ The effective charges of $\mathrm{Ag}$ and I are only 0.4 and -0.4 e, respectively. ${ }^{15}$ Specifically for $\mathrm{AgI}$, providing $\mathrm{Ag}^{+}$ions in water adjacent to $\mathrm{AgI}$ to achieve net zero surface charge increases the IN ability, ${ }^{26,34,35}$ indicating the importance of the electrostatic conditions near the surface.

Observation of the HB network of water molecules near AgI might provide insight into the way this substrate interacts with water molecules to facilitate ice nucleation. Surface-sensitive Xray photoemission spectroscopy (XPS) and Auger electron yield near-edge X-ray absorption fine structure (NEXAFS) spectroscopy at the oxygen K-edge are useful tools to probe the electronic structure and the local coordination of water molecules. ${ }^{36-41}$ XPS can provide surface-sensitive elemental information, the electronic state of an element, the thickness of an adsorbate layer, and depth information in the nanometer range, thus covering the adsorbate layer, the substrate surface, and the bulk underneath.

O K-edge NEXAFS spectroscopy probes the absorption of electro-magnetic radiation by excitation of core electrons into unoccupied molecular orbitals via dipole-induced transitions. The O K-edge NEXAFS spectra are very sensitive to the local $\mathrm{HB}$ network among the water molecules and thus they are arranged in a tetrahedral coordination as in ice or in a more disordered configuration as in liquid water. ${ }^{36,40,42,43}$ When collecting NEXAFS spectra through the detection of Auger electrons emitted during relaxation of the initial core hole, the method becomes surface-sensitive due to the low inelastic mean free path of the Auger electrons, similar to the case of XPS. Although non-linear optical methods may provide structural information as well, and even with higher surface sensitivity, ${ }^{23}$ the advantage of the NEXAFS-based method is the combination with XPS to have parallel chemical information. We have previously used this technique to detect changes to the HB structure of ice in the presence of ions. ${ }^{44-46}$ Following up from pioneering work by Verdaguer et al. who were the first to attempt at probing the structure of adsorbed water with this technique on $\mathrm{SiO}_{2}$, ${ }^{25}$ we further used it to observe liquid-like adsorbed water at the surface of $\mathrm{TiO}_{2}{ }^{17}$ This work aims to investigate the $\mathrm{HB}$ structure of adsorbed water molecules on the surface of AgI in situ while varying relative humidity under subsaturated conditions with respect to ice using XPS and electron yield O K-edge NEXAFS. Although this technique does not allow observing the nucleation of ice directly, it allows interrogating the adsorbed water in situ while in equilibrium with water vapor below saturation. We found that the adsorbed water on AgI particles shows a rather ice-like $\mathrm{HB}$ structure.

\section{MATERIALS AND EXPERIMENTAL METHODS}

The experiments were carried out at the in situ spectroscopy (ISS) beamline at the Swiss Light Source. Photoemission and electron yield NEXAFS spectra were acquired with a hemispherical electron analyzer coupled to a differentially pumped lens system (ScientaOmicron R4000/HiPP-2). The experimental cell (solid-gas interface chamber) under the conditions of the photoemission experiments has been described in a previous publication. ${ }^{47}$ More details of the sample configuration relevant for the present experiments are given in the Supporting Information.

The process chosen for the production of silver iodide particles was inspired by Nagare et al. ${ }^{48}$ Chemicals used for sample preparation were purchased from Sigma-Aldrich. We mixed $0.1 \mathrm{M}$ potassium iodide and $0.1 \mathrm{M}$ silver nitrate aqueous solutions and diluted it with pure water $(18.2 \mathrm{MOhm} \mathrm{cm}$ remaining conductivity), which led to the precipitation of yellowish AgI particles. The AgI product was then washed with pure water and centrifuged to separate the powder from the water. AgI particles were dried in an oven at $423 \mathrm{~K}$ for $3 \mathrm{~h}$ and then cooled down to room temperature at a rate of $0.3 \mathrm{~K} / \mathrm{min}$. Since the AgI is highly photosensitive, the sample was prepared in the absence of light to avoid its decomposition.

The synthesized AgI powder was dispersed in isopropanol and sonicated for $5 \mathrm{~min}$, forming a homogeneous suspension. Two $30 \mu \mathrm{L}$ droplets of the suspension were drop cast onto the surface of the sample holder, leaving a uniform layer of AgI powder on the surface of the sample holder. The sample was then introduced in the experimental cell. Water vapor was 
dosed to the cell by a capillary dosing system (see the Supporting Information, Section S1). Under isobaric conditions and by varying the temperature of the sample, we could effectively adjust the relative humidity at the sample surface, which leads to a varying amount of adsorbed water in equilibrium between the solid sample and water vapor. This method allows to keep the water vapor pressure constant for different relative humidities. This reduces the uncertainty in the retrieval of the spectra of adsorbed water. The experiments were repeated under three different conditions: (i) dry in high vacuum at $323 \mathrm{~K}$, (ii) $60 \%(251 \mathrm{~K}$ ), and (iii) $90 \%(247 \mathrm{~K}) \mathrm{RH}$ with respect to ice at $0.5 \mathrm{mbar}$ of water vapor pressure.

During the experiment, we made use of linearly polarized light at $0^{\circ}$. O 1s, I $3 \mathrm{~d}$, and $\mathrm{C} 1 \mathrm{~s}$ core level photoelectron spectra were acquired at photon energies such that each core level was obtained at the same five different kinetic energies, corresponding to five different probing depths. The corresponding excitation and kinetic energies are provided in the Supporting Information (Table S1). The probing depth is related to the inelastic mean free path $(\lambda)$ of the photoelectron (probing depth $=\lambda \cdot \cos \theta$, where $\theta$ is the electron take-off angle with respect to the surface normal). Photoemission peaks shown in this work were normalized to the photon flux, inelastic mean free path of photoelectrons, and the total photoionization cross section. A Shirley background was subtracted from the spectra before calculating the area. The binding energy scale of the measured core level electrons was

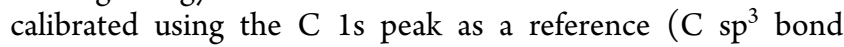
assigned to carbon contamination, at $284.8 \mathrm{eV}$ ). Peak fitting details are provided in the Supporting Information (Table S2). The O K-edge NEXAFS spectra were obtained by integrating the Auger KLL peak of oxygen within a kinetic energy window ranging from 425 to $525 \mathrm{eV}$, while scanning the photon energy from 527 to $560 \mathrm{eV}$.

\section{RESULTS AND DISCUSSION}

Adsorption of Water on the Agl Surface. The number of water molecules reversibly adsorbed on the surface of AgI particles at equilibrium was monitored via the $\mathrm{O} 1 \mathrm{~s}$ photoemission signal intensity excited by $870 \mathrm{eV}$ photons, as a function of relative humidity (Figure 1). Note that the equilibration time scale for water adsorption is in the order of nanoseconds. Thus, the desorption and adsorption steady state was maintained during the acquisition of spectra, and it is reasonable to assume that the adsorbed water layer on the AgI surface was not entirely homogeneous but was probably affected by the structure of the powder, edges, and defects, as also discussed by Marcolli et al. ${ }^{26}$

For the photon energy chosen, photoelectrons had a kinetic energy of $340 \mathrm{eV}$ corresponding to a probe depth of $\mathrm{ca} .1 \mathrm{~nm}$ (the most surface sensitive mode of this work). Under dry conditions (Figure 1c), $323 \mathrm{~K}$ in vacuum, the only broad feature in the spectrum at around $532 \mathrm{eV}$ is assigned to oxygenated adventitious carbon species. Adventitious carbon contamination is inevitable for samples that have been exposed to the ambient environment before transfer into the sample chamber, and such carbon species may build up further during $\mathrm{X}$-ray irradiation at pressures higher than standard ultra-high vacuum. A survey XPS scan, which shows carbon on the surface, is reported in Figure S2. Representative highresolution $\mathrm{C}$ 1s spectra at both dry and humid conditions are provided in Figure S3. Note that under dry conditions, although the spectrum is dominated by reduced carbon

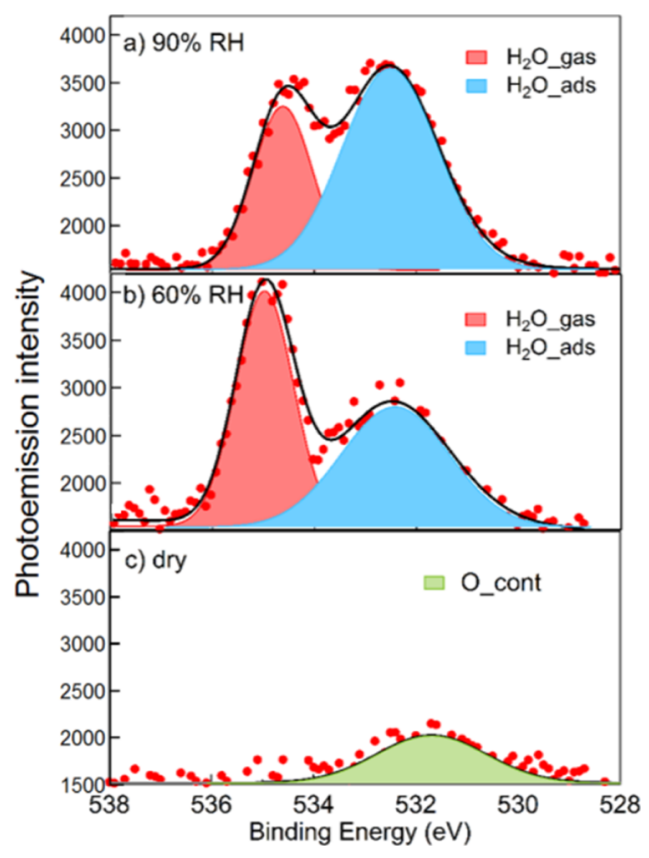

Figure 1. O 1s photoemission spectra measured with a photon energy of $870 \mathrm{eV}$ on the surface of AgI under vacuum (dry, $323 \mathrm{~K}$ ) (c), $60 \%$ $\mathrm{RH} / 251 \mathrm{~K}(\mathrm{~b})$, and $90 \% \mathrm{RH} / 247 \mathrm{~K}$ (a) conditions. The shaded areas indicate the components of a Gaussian peak deconvolution for $\mathrm{O} 1 \mathrm{~s}$ originating from $\mathrm{H}_{2} \mathrm{O}$ _gas, $\mathrm{H}_{2} \mathrm{O}$ _ads, and $\mathrm{O}_{-}$cont, respectively, as explained in the text. The three spectra share the vertical axis scale.

species, a feature corresponding to oxygenated carbon is present at $286.3 \mathrm{eV}$. Similar contaminating species have been reported in other studies. ${ }^{49-52}$ Figure 1 shows that, upon water dosing into the experimental chamber $\left(\mathrm{H}_{2} \mathrm{O}\right.$ partial pressure of $0.5 \mathrm{mbar}$ ), a sharp feature in the $\mathrm{O} 1 \mathrm{~s}$ at $535.0 \mathrm{eV}$, assigned to gas phase water, is observed at both RHs. Apart from hitting the AgI sample itself, the X-ray beam also overlaps with the gas phase just above the sample and within the acceptance volume of the analyzer electron optics. The binding energy obtained for gas phase water is not well defined since the binding energy calibration is based on the C 1s surface contamination (see below), which corrects for sample charging. Gas phase molecules experience a different potential as they are located between the sample surface and the grounded electron sampling aperture. As the relative humidity increases (Figure $1, \mathrm{~b}$ and a), a new broad feature appears at $532.5 \mathrm{eV}$, whose relative intensity is higher at $90 \% \mathrm{RH}$ than at $60 \% \mathrm{RH}$, which we attribute to adsorbed water molecules. ${ }^{53}$ The significant full width at half-maximum (see Table S2) of this peak results from different adsorption configurations, different adsorption sites exposed on the surface of a powder, and from the intermolecular interactions among the water molecules. The contribution of the contaminating component identified under dry conditions to the $\mathrm{O} 1 \mathrm{~s}$ cannot be assessed anymore. In more detail, Figure S3 shows that more of the oxidized carbonaceous species (e.g., carboxyls) appear at 90\% RH. The contribution of carboxyls to the $\mathrm{O} 1 \mathrm{~s}$ spectrum overlaps strongly with that of adsorbed water; ${ }^{53}$ thus, we refrained from fitting oxygenated carbon species to the $\mathrm{O}$ 1s under humid conditions due to the large uncertainty. Based on the $\mathrm{C} 1 \mathrm{~s}$ spectra (Supporting Information, Figure S3), the contribution of carboxyl carbon is about an order of magnitude less than that of total carbon. Therefore, in spite of substantial amounts of total carbon (see quantitative information from application 
of attenuation model below), the adsorbed water still dominates over oxygenated adventitious carbon species. Throughout this work, we deconvoluted all spectra into three Gaussian peaks (see Figure 1): oxygen from gas phase water $\left(\mathrm{H}_{2} \mathrm{O} \_\right.$gas, red peak $)$, adsorbed water $\left(\mathrm{H}_{2} \mathrm{O}\right.$ ads, blue peak), and oxygen belonging to the oxygenated carbon species (O_cont, green peak) under dry conditions.

Figure 2 shows the evolution of the I $3 \mathrm{~d}$ signal intensity under dry and 60 and $90 \% \mathrm{RH}$ at $0.5 \mathrm{mbar}$. The I 3d spectral

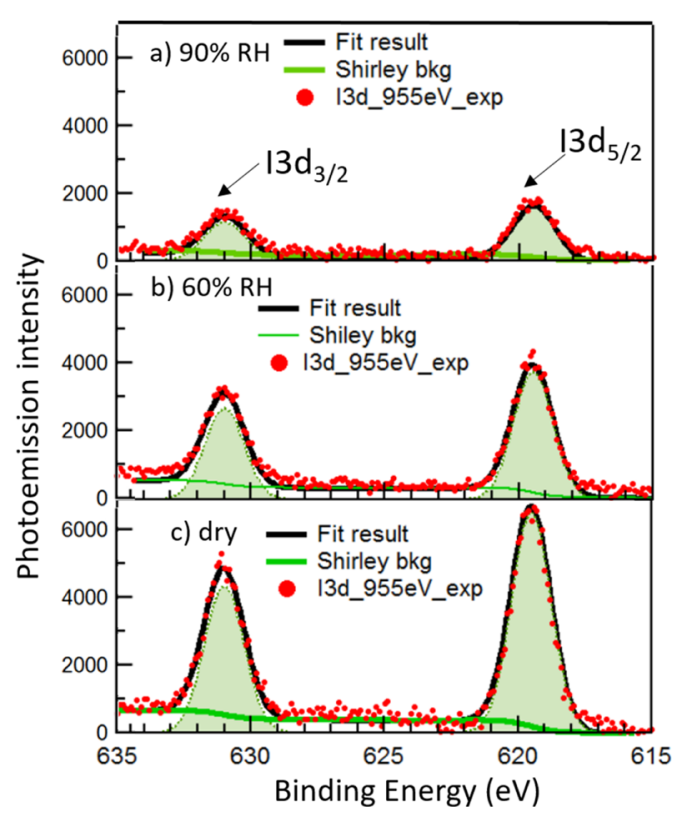

Figure 2. Plot of measured I 3d XPS spectra at dry (c), 60\% RH (b), and $90 \% \mathrm{RH}$ (a) with $h \nu=955 \mathrm{eV}$, corresponding to a kinetic energy of $340 \mathrm{eV}$. The S.O.S of I 3d is $11.5 \mathrm{eV}$. Gaussian deconvolutions of the I $3 \mathrm{~d}$ peak are shown by green shaded areas. The three spectra share the same vertical axis scale.

deconvolution by Gaussian peaks is also shown (shaded areas). The measured binding energies of $\mathrm{I} 3 \mathrm{~d}_{5 / 2}$ and $\mathrm{I} 3 \mathrm{~d}_{3 / 2}$ are 619.5 and $631.0 \mathrm{eV}$, respectively, with a spin-orbit split of $11.5 \mathrm{eV}$, in agreement with other literature reports for AgI. ${ }^{54,55}$ The fitting parameters are reported in the Supporting Information, Table S2. The increasing thickness of the adsorbed water layer on the AgI surface leads to an increasing attenuation of the I $3 \mathrm{~d}$ signal, as directly apparent from Figure 2 . Silver iodide is a photosensitive material, and while exposed to the synchrotron radiation, it may undergo decomposition. However, Figure 2 shows that the line shape of I $3 \mathrm{~d}$ does not evolve as a function of $\mathrm{RH}$, indicating that the chemical state change of iodine is negligible, within uncertainty and detection limit, during the time scale of the acquisition of a spectrum (about $5 \mathrm{~min}$ ). The same result was observed for silver (Ag $3 \mathrm{~d}$ spectra reported in Figure S4). The absence of new peak components in $\mathrm{Ag} \mathrm{3d}$ and I $3 \mathrm{~d}$ spectra also indicates that no partial solvation of iodide anions and silver cations occurred at the interface, as observed for more soluble salts in previous work. ${ }^{56}$

Depth Profile. To get a depth profile of the material under different conditions, the signals of $\mathrm{O} 1 \mathrm{~s}$ and I $3 \mathrm{~d}$ were acquired with increasing kinetic energy: $340 \mathrm{eV}$ (already discussed in the previous section), 550, 650, 750, and $900 \mathrm{eV}$. The signal of I $3 \mathrm{~d}$ from bulk $\mathrm{AgI}$ served as a reference to represent the bulk substrate and normalize the $\mathrm{O} 1 \mathrm{~s}$ photoemission signal intensity of adsorbed water. $\mathrm{O} 1 \mathrm{~s}\left(\mathrm{H}_{2} \mathrm{O} \_\right.$ads)/I 3d (AgI) ratios evaluated at 60 and $90 \% \mathrm{RH}$ are plotted as a function of the kinetic energy in Figure 3. When reporting the photo-

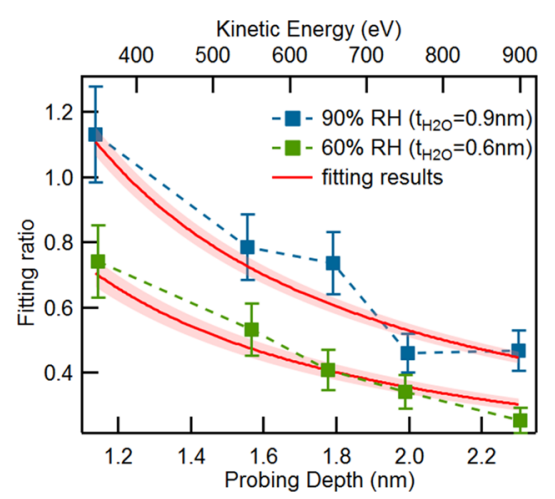

Figure 3. Fitting ratio of the photoemission intensity of $\mathrm{O} 1 \mathrm{~s}$ from adsorbed water to that of I $3 \mathrm{~d}$ measured at five different probing depths, under $60 \% \mathrm{RH}$ (green) and $90 \% \mathrm{RH}$ (blue). The solid red line represents the fitting results using the attenuation model described in the text and in the Supporting Information, with one standard deviation shown by the red shaded area.

emission intensity ratios between two elements at the same kinetic energy, those factors contributing to the measured intensity cancel, which are related to the analyzer transmission and/or depend on the measurement geometry of the experimental setup. As the probing depth increases, the relative contribution of the I $3 \mathrm{~d}$ signal intensity from the bulk starts to increase, while the contribution of oxygen atoms belonging to water adsorbed on the surface remains almost constant, leading to a general decrease of $\mathrm{O} 1 \mathrm{~s}\left(\mathrm{H}_{2} \mathrm{O} \_\right.$ads $) / \mathrm{I}$ 3d (AgI).

An attenuation model was developed to fit the kinetic energy-dependent signal intensity ratios of $\mathrm{O} 1 \mathrm{~s}\left(\mathrm{H}_{2} \mathrm{O}\right.$ ads $)$ to I 3d (AgI) and C 1s/I 3d (AgI). This model considers the surface being covered by a homogeneous layer of adsorbed water (see Section S4 in the Supporting Information). On top of this water layer, patchy areas of floating carbon islands with an average thickness, $t_{\mathcal{c}}$, cover a fraction $\gamma$ of the surface area, similar to a model proposed by Fadley. ${ }^{57}$ The equations used to calculate signal intensities of $\mathrm{O} 1 \mathrm{~s}$, I $3 \mathrm{~d}$, and $\mathrm{C} 1 \mathrm{~s}$ and the corresponding ratios are provided in the Supporting Information (Figures S7 and S8). The best fit resulted in a $\gamma$ of 0.80 and a carbon island thickness of $0.3 \mathrm{~nm}$ under dry conditions. Under humid conditions, at $60 \% \mathrm{RH}$, the water layer thickness is determined to be $0.6 \mathrm{~nm}$ (corresponding to approximately $2 \mathrm{~mL}$ of water), and the corresponding carbon island thickness is $0.6 \mathrm{~nm}$ with a $\gamma$ value of 0.6 . At $90 \%$, the water layer thickness is $0.9 \mathrm{~nm}$ (corresponding to approximately $3 \mathrm{~mL}$ of water) and the fractional coverage of carbon is further increased to 0.8 with an average carbon island thickness of $1.1 \mathrm{~nm}$. The uncertainty related to the fits are included in Figure S8. The results of our depth profile analysis suggest that $t_{\mathrm{c}}$ and $\gamma$ change with the RH. Higher RH conditions favor the growth of adventitious carbon islands. This implies that even though liquid water used as the source of vapor was purified by several freeze-pump-thaw cycles, it is inevitable to co-dose small amounts of carbon-containing species that are decomposed by the beam. This may partly derive from displacement of contaminating gases previously adsorbed at the chamber walls. $^{47,52}$ 
Structure of water adsorbed on Agl. In the discussion of NEXAFS spectra of adsorbed water and the relationship to the $\mathrm{HB}$ structure, we mainly rely on the comparison to the cases of liquid water and ice. In the Supporting Information, Figure S9, we show partial electron yield O K-edge NEXAFS spectra of liquid water, ice, and gas phase water. In the latter, three well-separated peaks represent the excitations from $\mathrm{O} 1 \mathrm{~s}$ into the $4 a_{1}(534 \mathrm{eV}), 2 b_{1}(536 \mathrm{eV})$, and Rydberg $(537 \mathrm{eV})$ orbitals of free water molecules. In the condensed phase (see also Figure 4a), these transitions strongly broaden and shift

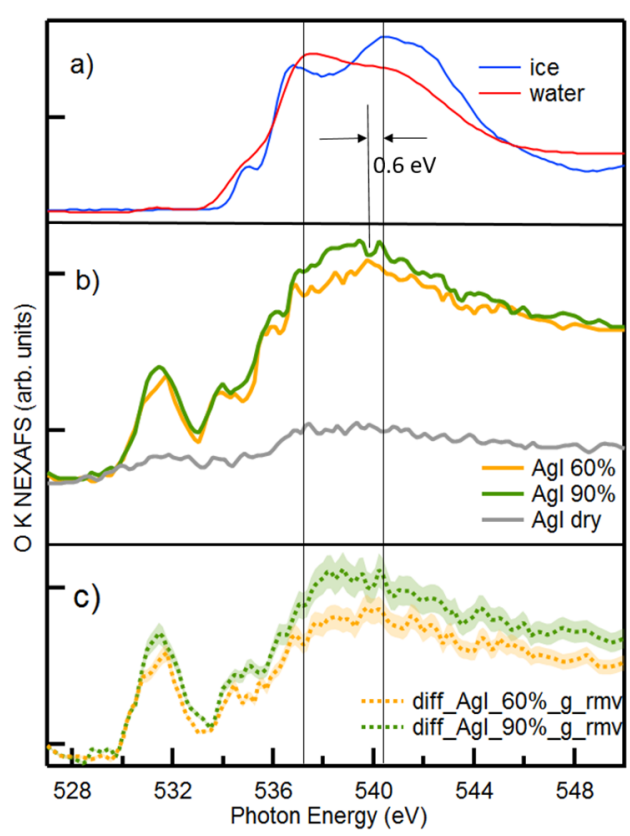

Figure 4. (a) Auger electron yield O K-edge NEXAFS spectra of liquid water and ice for comparison (also shown in the Supporting Information). (b) Electron yield O K-edge NEXAFS spectra from AgI particles under dry conditions (gray, $323 \mathrm{~K}$ ), 60\% RH (blue, at 251 $\mathrm{K}$ ), and $90 \% \mathrm{RH}$ (red, at $247 \mathrm{~K}$ ) at 0.5 mbar. (c) Difference spectra obtained by subtracting the spectrum measured at dry conditions and water gas phase from those under humid conditions shown in (b). Spectra of wet conditions (green and yellow lines in b) and their corresponding difference spectra (dashed green and yellow line in c), shown with different scales.

due to the close $\mathrm{O}-\mathrm{H}$ distance in $\mathrm{H}$-bonds in a range of configurations and strengths, ${ }^{38}$ which strongly affects the unoccupied orbitals in comparison to the gas phase case. In liquid water, strong intramolecular covalent $\mathrm{O}-\mathrm{H}$ bonds and asymmetric weak $\mathrm{H}$-bonding configurations prevail, leading to maximum absorption at around $537 \mathrm{eV}$ and a pronounced preedge at $535 \mathrm{eV}$. In contrast, in ice, the predominance of strong and symmetric tetrahedral configurations leads to maximum absorption at around 541-542 eV (Figure S9). Absorption at $535 \mathrm{eV}$ is attributed to free $\mathrm{OH}$ groups that are more prevalent in liquid water than in ice but also at the water-vapor interface. $^{38,58}$ In this work, we use the contrasting HB configurations embodied in the NEXAFS spectra of liquid water and ice as references to qualitatively describe the $\mathrm{HB}$ structure of adsorbed water on AgI. In the spectra of liquid water and ice, changes with temperature or between liquid and ice can be explained by the varying relative occurrence of different configurations rather than by energy shifts of the absorption features. ${ }^{59}$ The interaction between adsorbed water molecules and the solid surface might lead to shifts of absorption features, but the general shape and the appearance of the structural motives in the NEXAFS features is assumed be preserved. This was demonstrated for the liquid-like character of adsorbed water on $\mathrm{TiO}_{2}{ }^{17}$ and also for tetrahedrally coordinated water adsorbed on $\mathrm{Ru}(0001)$ once coverage reaches one monolayer and above. ${ }^{60}$

Figure $4 \mathrm{~b}$ shows the $\mathrm{O} \mathrm{K}$ edge of AgI samples under dry and humid conditions. The AgI spectrum measured under dry conditions (grey) shows only weak absorption features because the lattice of AgI is oxygen-free. The observed broad features likely result from the oxygen-containing carbonaceous contaminations, as confirmed by C 1s XPS and discussed in the previous section (Figure 1c). When the $\mathrm{RH}$ increases (yellow for $60 \% \mathrm{RH}$ at $251 \mathrm{~K}$ and green for $90 \% \mathrm{RH}$ at $247 \mathrm{~K}$ ), water adsorbs and starts to dominate the spectrum. At $60 \%$ $\mathrm{RH}$, the absorption features at $537 \mathrm{eV}$ and, especially, at 540 $\mathrm{eV}$ increase. As the $\mathrm{RH}$ increases to $90 \%$, the absorption features further increase. The spectra of water on AgI under humid conditions exhibit higher absorption feature at 540 than at $537 \mathrm{eV}$. Such a behavior is comparable to the case of ice, but with the absorption maximum slightly shifted (by approximately $0.6 \mathrm{eV}$ ) toward lower photon energy (shown in Figure 4). We also note a strongly increasing absorption at $532 \mathrm{eV}$, related to the oxygenated carbon contaminations. The $\mathrm{O} \mathrm{K}$ edge spectrum of typical carboxylic acids or ketones most likely formed under humid and more oxidizing conditions in the presence of water vapor, and detected in the C 1s spectra (Figure S3b), is characterized by an intense peak at $532 \mathrm{eV}$. It is the result of the transition of 1 s electrons of oxygen in carbonyl or carboxyl functions into $\pi^{*}$ unoccupied molecular orbitals. $^{61}$ Absorption between 537 and $545 \mathrm{eV}$ for such species is rather featureless and relatively weak. As also mentioned in the discussion of the $\mathrm{O}$ 1s photoemission spectra (Figure 1), their relative proportion can be considered rather small.

By subtracting the spectrum of dry AgI and that of the water gas phase from those acquired at different $\mathrm{RH}$ values, we focus exclusively on the adsorbed water layer (Figure 4c). Apart from $\mathrm{C}=\mathrm{O}$ absorption in the pre-edge, these difference spectra exhibit maximum intensity between 539 and $540 \mathrm{eV}$, where the stronger and more symmetric $\mathrm{H}$-bonds contribute, and lower intensity around $537 \mathrm{eV}$, where the characteristic absorptions for asymmetric weak $\mathrm{H}$-bonds predominate. This indicates that under subsaturated relative humidity conditions, water molecules adsorbed on AgI have a higher proportion of tetrahedrally and symmetrically coordinated water molecules. However, as mentioned above for the raw spectra, the maximum of the absorption band of the difference spectra are negatively shifted by approximately $0.6 \mathrm{eV}$ compared to the reference spectrum of ice. The local geometry of the substrate and of the adsorbate are possibly responsible for this apparent shift, which was also observed on other surfaces. ${ }^{17,60}$

To more quantitatively estimate the relative share of water molecules in liquid-like weak $\mathrm{H}$-bonding configurations versus those in tetrahedral ice-like configurations, all NEXAFS spectra were deconvoluted with Gaussian peaks. The results are presented in Figures S10 and S11. The deconvolution followed the rationale used by Cappa et al. ${ }^{62}$ and Nilsson et al..$^{38}$ in which the energy positions, widths, and number of peaks were not chosen to represent discrete transitions but rather to obtain a good overall fit of the spectrum. Still, we chose them in a similar way to allow an estimate of liquid- and ice-like 
adsorbed water (see the Supporting Information for more information). The results are summarized in Figure 5. The

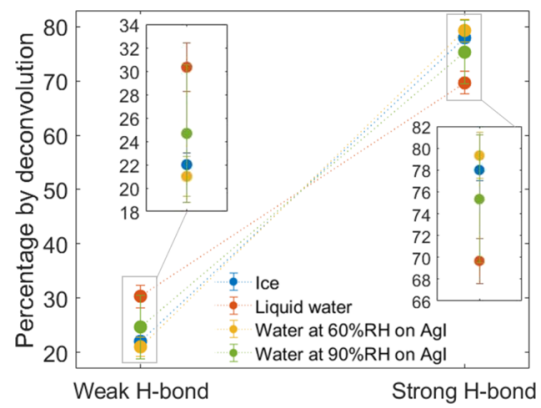

Figure 5. Fraction (\%) of the electron yield O K-edge NEXAFS peak areas attributed to configurations of adsorbed water molecules with weak $\mathrm{H}$-bonds and those with strong $\mathrm{H}$-bonds on the AgI surface at $60 \% \mathrm{RH}(0.5 \mathrm{mbar}, 251 \mathrm{~K})$ and $90 \% \mathrm{RH}(0.5 \mathrm{mbar}, 247 \mathrm{~K})$. For comparison, results for ice (blue) and liquid water (red) as references are also reported in the plot. The insets are shown to more clearly illustrate the differences among the samples.

weak asymmetric $\mathrm{H}$-bonds are represented by the sum of the areas of red peaks (peaks 2, 3, and 4 of the NEXAFS spectra, Figure S10) and the strong symmetric tetrahedral configurations by the sum of the areas of the blue peaks (peaks 5 and 6 , Figure S10). The total area of the blue peaks is always higher than that of the red peaks. The relative share of the red peaks is higher in liquid water than in ice (weak H-bond, left column in Figure 5), whereas the relative share of the blue peaks is higher in ice than in liquid water (strong H-bond, right column in Figure 5). Concerning water molecules adsorbed on the surface of AgI, at $60 \%$ (yellow) and 90\% RH (green), both shares of weak and strong H-bonds are closer to that for ice than to that for liquid water. Obviously, this analysis strongly depends on the fitting procedure, specifically on the numbers of peaks used to represent the whole absorption edge, and to the attribution of the peaks to the weak and strong H-bond category. In order to illustrate the uncertainty and to assess the sensitivity to the choice and attribution of the peaks, we have fitted all NEXAFS spectra also with just six Gaussians rather than seven and by once re-assigning one of the peaks to either the strong (blue) or the weak (red) H-bond category (see the Supporting Information). The result of this sensitivity analysis is included in Figure 5 as error bars. This underlines the robustness of our conclusion that at both relative humidities the HB configuration is closer to being ice-like than liquid-like. This seems more pronounced at $60 \% \mathrm{RH}$, when on average 2 $\mathrm{mL}$ of water adsorbed on $\mathrm{AgI}$ particles mostly contribute to the O K-edge NEXAFS signal. However, we would only expect a certain fraction of water molecules being able to attain a fully tetrahedral coordination as in ice because a substantial part is likely engaging in strong interaction with the substrate. We also note the significant uncertainty and would not like to overinterpret these fitting results. In turn, at $90 \% \mathrm{RH}$, a slightly lower share of tetrahedral coordination of water molecules is derived than at $60 \% \mathrm{RH}$. Thus, it does not seem that the formation of a completely hexagonal structure is limited by the amount of water molecules at the surface at equilibrium. Rather, we have to remain aware that even for the surface of bulk crystalline ice, the topmost surface layer is disordered, which manifests in changing relative proportions in the NEXAFS spectra. ${ }^{63,64}$ Nevertheless, this layer with at least partial abundance of tetrahedrally coordinated water molecules may facilitate the stabilization of ice embryos at saturation and above.

\section{CONCLUSIONS}

We have experimentally investigated the $\mathrm{HB}$ structure of adsorbed water on a AgI powder surface by using in situ XPS and electron yield NEXAFS spectroscopy techniques. The thickness of the equilibrium adsorbed water layer is determined at elevated $\mathrm{RH}$ with the help of an attenuation model that takes into account the presence of adventitious carbon in the form of islands at the topmost surface. On increasing the $\mathrm{RH}$ from dry conditions to 60 and $90 \%$, the thickness of the water layer increases from approximately 2 to $3 \mathrm{~mL}$, respectively. Our results are the first direct experimental evidence unraveling the local coordination of an $\mathrm{HB}$ structure on an actual and realistic AgI particle surface at a molecular level. The O K-edge NEXAFS spectra of water on the AgI surface generally show an enhanced high-energy absorption edge located at $540 \mathrm{eV}$, which is typically observed in the presence of tetrahedrally coordinated (ice-like) water molecules. This is also evident by the comparison with the $\mathrm{O} \mathrm{K}$ edge spectrum of ice.

AgI was reported ashydrophilic ${ }^{65,66}$ in spite of its very low solubility in water, ${ }^{33}$ probably because it may attract water molecules without perturbing their electronic structure by ion dissolution. When the coverage of water molecules is low, at $60 \% \mathrm{RH}$, a larger fraction of water molecules are adopting a tetrahedral, ice-like structure, highlighting the potential of AgI to promote ice nucleation. The combined results of XPS and NEXAFS suggest that water molecules adsorbed on the surface of solid AgI particles already start to display an ordered hydrogen bond structure at subsaturated conditions with respect to ice, which is mirrored in the role of AgI as an efficient ice nucleus. This is in contrast to the case of water adsorbed on $\mathrm{TiO}_{2}$ nanoparticles (known to be inefficient INs), in which surface water molecules are still liquid-like at a temperature close to that where homogeneous ice nucleation occurs. ${ }^{17}$ Thus, our work reveals how the water HB structure on a solid particle surface is the result of a complex interplay of interactions of water molecules among each other and with the substrates on a molecular level.

\section{ASSOCIATED CONTENT}

\section{Supporting Information}

The Supporting Information is available free of charge at https://pubs.acs.org/doi/10.1021/acs.jpcc.1c01767.

Experiment setup, measurement parameters, supporting XPS spectra, attenuation model and fitting of the $\mathrm{O}$ 1s_ads/I 3d ratio, patched carbon islands model and its fitting results, and $\mathrm{O}$ K-edge NEXAFS spectra and their Gaussian deconvolution (PDF)

\section{AUTHOR INFORMATION}

\section{Corresponding Author}

Markus Ammann - Laboratory of Environmental Chemistry, Paul Scherrer Institut, Villigen 5232, Switzerland; ○ orcid.org/0000-0001-5922-9000;

Email: markus.ammann@psi.ch 


\section{Authors}

Huanyu Yang - Laboratory of Environmental Chemistry, Paul Scherrer Institut, Villigen 5232, Switzerland; Institute of Atmospheric and Climate Science, ETH Zürich, Zürich 8092, Switzerland; 이이이.org/0000-0002-0927-9621

Anthony Boucly - Laboratory of Environmental Chemistry, Paul Scherrer Institut, Villigen 5232, Switzerland; () orcid.org/0000-0003-1436-3462

Jérôme Philippe Gabathuler - Laboratory of Environmental Chemistry, Paul Scherrer Institut, Villigen 5232, Switzerland; Institute of Atmospheric and Climate Science, ETH Zürich, Zürich 8092, Switzerland

Thorsten Bartels-Rausch - Laboratory of Environmental Chemistry, Paul Scherrer Institut, Villigen 5232, Switzerland; (1) orcid.org/0000-0002-7548-2572

Luca Artiglia - Laboratory of Environmental Chemistry and Laboratory for Catalysis and Sustainable Chemistry, Paul Scherrer Institut, Villigen 5232, Switzerland; (1) orcid.org/ 0000-0003-4683-6447

Complete contact information is available at:

https://pubs.acs.org/10.1021/acs.jpcc.1c01767

\section{Author Contributions}

Y.H., L.A., and M.A. designed the work and conceived the experiment; Y.H., J.G., A.B., and L.A. performed the XPS and NEXAFS spectroscopy measurements; Y.H. analyzed and interpreted the XPS and NEXAFS data with the help of M.A., L.A., and A.B.; T.B.R. adapted the cryo sample holder and contributed to the discussion of the NEXAFS spectra; Y.H, L.A., and M.A worked on the manuscript together. All authors contributed to the discussion and approved the final version of the manuscript.

\section{Notes}

The authors declare no competing financial interest.

\section{ACKNOWLEDGMENTS}

This work was performed at the ISS beamline of the Swiss Light Source, Paul Scherrer Institute. We acknowledge the technical support by Andrés Laso and support by the beamline staff Dr. Jörg Raabe. This work was supported by the Swiss National Science Foundation (grant no. 169176).

\section{ABBREVIATIONS}

XPS X-ray photoemission spectroscopy

NEXAFS near-edge X-ray fine structure

\section{REFERENCES}

(1) Borduas, N.; Donahue, N. M. The Natural Atmosphere. In Green Chemistry; Török, B., Dransfield, T., Eds.; Elsevier, 2018, Chapter 3.1, pp 131-150. DOI: 10.1016/b978-0-12-809270-5.00006-6

(2) Cantrell, W.; Heymsfield, A. Production of Ice in Tropospheric Clouds: A Review. Bull. Am. Meteorol. Soc. 2005, 86, 795-808.

(3) Abbatt, J. P. D. Interactions of Atmospheric Trace Gases with Ice Surfaces: Adsorption and Reaction. Chem. Rev. 2003, 103, 47834800.

(4) Wang, P. K. Physics and Dynamics of Clouds and Precipitation; Cambridge University Press: Cambridge, 2013.

(5) Hoose, C.; Möhler, O. Heterogeneous Ice Nucleation on Atmospheric Aerosols: A Review of Results from Laboratory Experiments. Atmos. Chem. Phys. 2012, 12, 9817-9854.

(6) Marcolli, C. Deposition Nucleation Viewed as Homogeneous or Immersion Freezing in Pores and Cavities. Atmos. Chem. Phys. 2014, 14, 2071-2104.
(7) Kanji, Z. A.; Ladino, L. A.; Wex, H.; Boose, Y.; Burkert-Kohn, M.; Cziczo, D. J.; Krämer, M. Overview of Ice Nucleating Particles. Meteorol. Monogr. 2017, 58, 1.1-1.33.

(8) Pandey, R.; et al. Ice-Nucleating Bacteria Control the Order and Dynamics of Interfacial Water. Sci Adv. 2016, 2, No. e1501630.

(9) Maccarini, M. Water at Solid Surfaces: A Review of Selected Theoretical Aspects and Experiments on the Subject. Biointerphases 2007, 2, MR1-MR15.

(10) Sosso, G. C.; Tribello, G. A.; Zen, A.; Pedevilla, P.; Michaelides, A. Ice Formation on Kaolinite: Insights from Molecular Dynamics Simulations. J. Chem. Phys. 2016, 145, 211927.

(11) Hu, X. L.; Michaelides, A. Water on the Hydroxylated (001) Surface of Kaolinite: From Monomer Adsorption to a Flat 2d Wetting Layer. Surf. Sci. 2008, 602, 960-974.

(12) Lupi, L.; Molinero, V. Does Hydrophilicity of Carbon Particles Improve Their Ice Nucleation Ability? J. Phys. Chem. A 2014, 118, $7330-7337$.

(13) Qiu, Y.; Lupi, L.; Molinero, V. Is Water at the Graphite Interface Vapor-Like or Ice-Like? J. Phys. Chem. B 2018, 122, 36263634.

(14) Shevkunov, S. V. Computer Simulation of Water Vapor Adsorption on the Surface of a Crystal B-AgI Regular Shape Nanoparticle. Prot. Met. Phys. Chem. Surf. 2019, 55, 41-49.

(15) Ward, R. C.; Holdman, J. M.; Hale, B. N. Monte Carlo Studies of Water Monolayer Clusters on Substrates: Hexagonal AgI. J. Chem. Phys. 1982, 77, 3198-3202.

(16) Kiselev, A.; Bachmann, F.; Pedevilla, P.; Cox, S. J.; Michaelides, A.; Gerthsen, D.; Leisner, T. Active Sites in Heterogeneous Ice Nucleation-the Example of K-Rich Feldspars. Science 2017, 355, 367-371.

(17) Orlando, F.; Artiglia, L.; Yang, H.; Kong, X.; Roy, K.; Waldner, A.; Chen, S.; Bartels-Rausch, T.; Ammann, M. Disordered Adsorbed Water Layers on $\mathrm{TiO}_{2}$ Nanoparticles under Subsaturated Humidity Conditions at 235 K. J. Phys. Chem. Lett. 2019, 10, 7433-7438.

(18) Anderson, B. J.; Hallett, J. Supersaturation and Time Dependence of Ice Nucleation from the Vapor on Single Crystal Substrates. J. Atmos. Sci. 1976, 33, 822-832.

(19) Vonnegut, B. The Nucleation of Ice Formation by Silver Iodide. J. Appl. Phys. 1947, 18, 593-595.

(20) Zielke, S. A.; Bertram, A. K.; Patey, G. N. A Molecular Mechanism of Ice Nucleation on Model AgI Surfaces. J. Phys. Chem. B 2015, 119, 9049-9055.

(21) Pedevilla, P.; Cox, S. J.; Slater, B.; Michaelides, A. Can Ice-Like Structures Form on Non-Ice-Like Substrates? The Example of the KFeldspar Microcline. J. Phys. Chem. C 2016, 120, 6704-6713.

(22) Abdelmonem, A.; Backus, E. H. G.; Hoffmann, N.; Sánchez, M. A.; Cyran, J. D.; Kiselev, A.; Bonn, M. Surface-Charge-Induced Orientation of Interfacial Water Suppresses Heterogeneous Ice Nucleation on $\alpha$-Alumina (0001). Atmos. Chem. Phys. 2017, 17, $7827-7837$.

(23) Abdelmonem, A.; Lützenkirchen, J.; Leisner, T. Probing IceNucleation Processes on the Molecular Level Using Second Harmonic Generation Spectroscopy. Atmos. Meas. Tech. 2015, 8, 3519-3526.

(24) Abdelmonem, A.; Ratnayake, S.; Toner, J. D.; Lützenkirchen, J. Cloud History Can Change Water-Ice-Surface Interactions of Oxide Mineral Aerosols: A Case Study on Silica. Atmos. Chem. Phys. 2020, 20, 1075-1087.

(25) Verdaguer, A.; Weis, C.; Oncins, G.; Ketteler, G.; Bluhm, H.; Salmeron, M. Growth and Structure of Water on $\mathrm{SiO}_{2}$ Films on $\mathrm{Si}$ Investigated by Kelvin Probe Microscopy and in Situ X-Ray Spectroscopies. Langmuir 2007, 23, 9699-9703.

(26) Marcolli, C.; Nagare, B.; Welti, A.; Lohmann, U. Ice Nucleation Efficiency of AgI: Review and New Insights. Atmos. Chem. Phys. 2016, 16, 8915-8937.

(27) Silverman, B. A. A Critical Assessment of Glaciogenic Seeding of Convective Clouds for Rainfall Enhancement. Bull. Am. Meteorol. Soc. 2001, 82, 903-923. 
(28) Silver Iodide (AgI) Crystal Structure, High Pressure Modifications, Lattice Parameters. II-VI and I-VII Compounds; Madelung, O., Rössler, U., Schulz, M., Eds.; Springer-Verlag Berlin Heidelberg: Springermaterials, 1999; Vol. 41b, DOI: 10.1007/ 10681719_130.

(29) Fortes, A. D. Accurate and Precise Lattice Parameters of $\mathrm{H}_{2} \mathrm{O}$ and $\mathrm{D}_{2} \mathrm{O}$ Ice Ih between 1.6 and $270 \mathrm{~K}$ from High-Resolution Timeof-Flight Neutron Powder Diffraction Data. Acta Crystallogr., Sect. B: Struct. Sci., Cryst. Eng. Mater. 2018, 74, 196-216.

(30) Conrad, P.; Ewing, G. E.; Karlinsey, R. L.; Sadtchenko, V. Ice Nucleation on $\mathrm{BaF}_{2}(111)$. J. Chem. Phys. 2005, 122, 064709.

(31) Head, R. B. Steroids as Ice Nucleators. Nature 1961, 191, $1058-1059$.

(32) Nutt, D. R.; Stone, A. J. Adsorption of Water on the $\mathrm{BaF}_{2}(111)$ Surface. J. Chem. Phys. 2002, 117, 800-807.

(33) Grünewald, H. Solubilities of Inorganic and Organic Compounds. Herausgeg. v. H. Stephen und T. Stephen. Pergamon Press, Oxford-London-New York-Paris 1963. 1. engl. Aufl., Vol. 1, Part I: 960 S., geb. $£ 10.0 .0$. Vol. 1, Part II: 970 S., geb. $£ 12.10 .0$. Angew. Chem. 1964, 76, 832.

(34) Edwards, G. R.; Evans, L. F. Effect of Surface Charge on Ice Nucleation by Silver Iodide. J. Chem. Soc., Faraday Trans. 1962, 58, 1649-1655.

(35) Fletcher, N. H. Entropy Effect in Ice Crystal Nucleation. J. Chem. Phys. 1959, 30, 1476-1482.

(36) Cavalleri, M.; Ogasawara, H.; Pettersson, L. G. M.; Nilsson, A. The Interpretation of X-Ray Absorption Spectra of Water and Ice. Chem. Phys. Lett. 2002, 364, 363-370.

(37) Fransson, T.; Harada, Y.; Kosugi, N.; Besley, N. A.; Winter, B.; Rehr, J. J.; Pettersson, L. G. M.; Nilsson, A. X-Ray and Electron Spectroscopy of Water. Chem. Rev. 2016, 116, 7551-7569.

(38) Nilsson, A.; et al. X-Ray Absorption Spectroscopy and X-Ray Raman Scattering of Water and Ice; an Experimental View. J. Electron Spectrosc. Relat. Phenom. 2010, 177, 99-129.

(39) Schiros, T.; Andersson, K. J.; Pettersson, L. G. M.; Nilsson, A.; Ogasawara, H. Chemical Bonding of Water to Metal Surfaces Studied with Core-Level Spectroscopies. J. Electron Spectrosc. Relat. Phenom. 2010, 177, 85-98.

(40) Wilson, K. R.; Cavalleri, M.; Rude, B. S.; Schaller, R. D.; Nilsson, A.; Pettersson, L. G. M.; Goldman, N.; Catalano, T.; Bozek, J. D.; Saykally, R. J. Characterization of Hydrogen Bond Acceptor Molecules at the Water Surface Using near-Edge X-Ray Absorption Fine-Structure Spectroscopy and Density Functional Theory. J. Phys. Condens. Matter 2002, 14, L221.

(41) Winter, B.; Faubel, M. Photoemission from Liquid Aqueous Solutions. Chem. Rev. 2006, 106, 1176-1211.

(42) Smith, J. D.; Cappa, C. D.; Wilson, K. R.; Messer, B. M.; Cohen, R. C.; Saykally, R. J. Energetics of Hydrogen Bond Network Rearrangements in Liquid Water. Science 2004, 306, 851-853.

(43) Myneni, S.; et al. Spectroscopic Probing of Local HydrogenBonding Structures in Liquid Water. J. Phys.: Condens. Matter 2002, 14, L213.

(44) Kong, X.; Waldner, A.; Orlando, F.; Artiglia, L.; Huthwelker, T.; Ammann, M.; Bartels-Rausch, T. Coexistence of Physisorbed and Solvated $\mathrm{HCl}$ at Warm Ice Surfaces. J. Phys. Chem. Lett. 2017, 8, 4757-4762.

(45) Bartels-Rausch, T.; Orlando, F.; Kong, X.; Artiglia, L.; Ammann, M. Experimental Evidence for the Formation of Solvation Shells by Soluble Species at a Nonuniform Air-Ice Interface. ACS Earth Space Chem. 2017, 1, 572-579.

(46) Waldner, A.; Artiglia, L.; Kong, X.; Orlando, F.; Huthwelker, T.; Ammann, M.; Bartels-Rausch, T. Pre-Melting and the Adsorption of Formic Acid at the Air-Ice Interface at $253 \mathrm{~K}$ as Seen by NEXAFS and XPS. Phys. Chem. Chem. Phys. 2018, 20, 24408-24417.

(47) Orlando, F.; et al. The Environmental Photochemistry of Oxide Surfaces and the Nature of Frozen Salt Solutions: A New in Situ Xps Approach. Top. Catal. 2016, 59, 591-604.
(48) Nagare, B.; Marcolli, C.; Stetzer, O.; Lohmann, U. Comparison of Measured and Calculated Collision Efficiencies at Low Temperatures. Atmos. Chem. Phys. 2015, 15, 13759-13776.

(49) Mangolini, F.; McClimon, J. B.; Rose, F.; Carpick, R. W. Accounting for Nanometer-Thick Adventitious Carbon Contamination in X-Ray Absorption Spectra of Carbon-Based Materials. Anal. Chem. 2014, 86, 12258-12265.

(50) Landoulsi, J.; Genet, M. J.; Fleith, S.; Touré, Y.; Liascukiene, I.; Méthivier, C.; Rouxhet, P. G. Organic Adlayer on Inorganic Materials: XPS Analysis Selectivity to Cope with Adventitious Contamination. Appl. Surf. Sci. 2016, 383, 71-83.

(51) Stipp, S. L.; Hochella, M. F. Structure and Bonding Environments at the Calcite Surface as Observed with X-Ray Photoelectron Spectroscopy (XPS) and Low Energy Electron Diffraction (LEED). Geochim. Cosmochim. Acta 1991, 55, 1723-1736.

(52) Trotochaud, L.; et al. Water Adsorption and Dissociation on Polycrystalline Copper Oxides: Effects of Environmental Contamination and Experimental Protocol. J. Phys. Chem. B 2018, 122, 10001008.

(53) Ketteler, G.; Ashby, P.; Mun, B. S.; Ratera, I.; Bluhm, H.; Kasemo, B.; Salmeron, M. In Situ Photoelectron Spectroscopy Study of Water Adsorption on Model Biomaterial Surfaces. J. Phys.: Condens. Matter 2008, 20, 184024.

(54) Kaushik, V. K. XPS core level spectra and Auger parameters for some silver compounds. J. Electron Spectrosc. Relat. Phenom. 1991, 56, 273-277.

(55) Moers, H.; Klewe-Nebenius, H.; Pfennig, G.; Ache, H. J. Untersuchungen zur Wechselwirkung von gasförmigem molekularem Iod mit Silberoberflächen mittels XPS und AES. Fresenius Z. Anal. Chem. 1987, 329, 361-366.

(56) Verdaguer, A.; Sacha, G. M.; Bluhm, H.; Salmeron, M. Molecular Structure of Water at Interfaces: Wetting at the Nanometer Scale. Chem. Rev. 2006, 106, 1478-1510.

(57) Fadley, C. S.; Baird, R. J.; Siekhaus, W.; Novakov, T.; Bergström, S. Å. L. Surface Analysis and Angular Distributions in XRay Photoelectron Spectroscopy. J. Electron Spectrosc. Relat. Phenom. 1974, 4, 93-137.

(58) Wernet, P.; et al. The Structure of the First Coordination Shell in Liquid Water. Science 2004, 304, 995-999.

(59) Nilsson, A.; Pettersson, L. G. M. The Structural Origin of Anomalous Properties of Liquid Water. Nat. Commun. 2015, 6, 8998.

(60) Nordlund, D.; Ogasawara, H.; Andersson, K. J.; Tatarkhanov, M.; Salmerón, M.; Pettersson, L. G. M.; Nilsson, A. Sensitivity of XRay Absorption Spectroscopy to Hydrogen Bond Topology. Phys. Rev. B: Condens. Matter Mater. Phys. 2009, 80, 233404.

(61) Horikawa, Y.; Arai, H.; Tokushima, T.; Shin, S. Spectral Fingerprint in X-Ray Absorption for Hydrogen-Bonded Dimer Formation of Acetic Acids in Solution. Chem. Phys. Lett. 2012, 522, $33-37$.

(62) Cappa, C. D.; Smith, J. D.; Wilson, K. R.; Messer, B. M.; Gilles, M. K.; Cohen, R. C.; Saykally, R. J. Effects of Alkali Metal Halide Salts on the Hydrogen Bond Network of Liquid Water. J. Phys. Chem. B 2005, 109, 7046-7052.

(63) Bluhm, H.; Hävecker, M.; Knop-Gericke, A.; Kiskinova, M.; Schlögl, R.; Salmeron, M. In Situ X-Ray Photoelectron Spectroscopy Studies of Gas-Solid Interfaces at near-Ambient Conditions. MRS Bull. 2011, 32, 1022-1030.

(64) Křepelová, A.; Huthwelker, T.; Bluhm, H.; Ammann, M. Surface Chemical Properties of Eutectic and Frozen NaCl Solutions Probed by XPS and NEXAFS. ChemPhysChem 2010, 11, 3859-3866.

(65) Parungo, F. Physical and Chemical Properties of AgIAerosols Formed at Simulated Airborne Conditions; Department of Commerce, National Oceanic and Atmospheric Administration, 1975.

(66) De Pena, R. G.; Caimi, E. A. Hygroscopicity and Chemical Composition of Silver Iodide Smoke Used in Cloud Seeding Experiments. J. Atmos. Sci. 1967, 24, 383-386. 